\title{
PERTURBATION THEORY FOR LYAPUNOV EXPONENTS OF A TORAL MAP: EXTENSION OF A RESULT OF SHUB AND WILKINSON.
}

\author{
by David Ruelle*.
}

\begin{abstract}
Starting from a hyperbolic toral automorphism, we obtain, for a small volume preserving perturbation, an exact and rigorous second order perturbation expansion of the Lyapunov exponents.
\end{abstract}

Keywords: Lyapunov exponent, toral automorphism, hyperbolicity.

\footnotetext{
* Mathematics Dept., Rutgers University, and IHES. 91440 Bures sur Yvette, France. $<$ ruelle@ihes.fr $>$
} 
We consider volume preserving perturbations $F$ of a diffeomorphism $F_{0}=(\Phi, J)$ of $\mathbf{T}^{m+1}=\mathbf{T}^{m} \times \mathbf{T}$, where $\Phi$ is a hyperbolic automorphism of $\mathbf{T}^{m}$, and $J$ is a translation of $\mathbf{T}$. Writing $F=F_{0}+a F^{\prime}$, we shall show that the Lyapunov exponents for ( $F$, volume) can be expanded to second order in $a$ (Theorem 1). In particular, the central Lyapunov exponent $\lambda^{c}$ of $(F$, volume), to second order in $a$, is generally $\neq 0$ (Corolary 11). For a special family of perturbations one obtains particularly simple formulae, first noted by Shub and Wilkinson [10]. We recover their result in Theorem 12. We deviate from [10] mostly in that we don't have differentiability of $\lambda^{c}$, only a second order expansion around $a=0$. The ideas used here are largely those in Shub and Wilkinson [10], and can be appreciated in the background provided by Hirsh, Pugh and Shub [6], Burns and Wilkinson [3], Ruelle and Wilkinson [9]. For recent work related to Lyapunov exponents, see also Bonatti, Gómez-Mont and Viana [2], Avila and Bochi [1].

After completing the writing of this paper, the author received a preprint by D. Dolgopyat [4], which develops similar ideas in a more general setting, but without the specific formulas we obtain here.

\section{Theorem.}

Let $\Phi$ be a hyperbolic automorphism of $\mathbf{T}^{m}$, and $J: y \mapsto y+\alpha(\bmod 1)$ a translation of $\mathbf{T}$. Define $F_{0}=(\Phi, J)$, and let $F=F_{0}+a F^{\prime}$ be a $C^{2}$ perturbation of $F_{0}$, volume preserving to first order in $a$. (We take $F^{\prime}: \mathbf{T}^{m+1} \mapsto \mathbf{R}^{m+1}$ and $F_{0} \xi+a F^{\prime}(\xi)$ has to be understood (mod 1$)$ in each component). Let $\lambda_{1}<\lambda_{2}<\ldots$ be the Lyapunov exponents of $\left(F_{0}\right.$, volume) and $m_{1}, m_{2}, \ldots$ their multiplicities (the exponent $=0$ occurs with multiplicity 1). Also let $\lambda_{a}^{(1)} \leq \lambda_{a}^{(2)} \leq \ldots$ be the Lyapunov exponents of ( $F$, volume) repeated according to multiplicity. Then we have the second order expansion

$$
\sum_{\ell=m_{1}+\ldots+m_{r-1}+1}^{m_{1}+\ldots+m_{r}} \lambda_{a}^{(\ell)}=m_{r} \lambda_{r}+a^{2} L_{r}+o\left(a^{2}\right)
$$

If $m_{r}=1$, and writing $\lambda_{r}=\lambda_{0}^{(\ell)}$, we have

$$
\lambda_{a}^{(\ell)}=\lambda_{0}^{(\ell)}+a^{2} L^{(\ell)}+o\left(a^{2}\right)
$$

(this applies in particular to $\lambda^{c}=\lambda_{a}^{(\ell)}$ for $\lambda_{0}^{(\ell)}=0$ ).

An explicit expression for $L_{r}$ can be obtained (see Proposition 9). We do not assume ergodicity of ( $F$, volume), and therefore we use integrated Lyapunov exponents (averaged over the volume), see however Remark 15(a).

If one likes one may to $F_{0}+a F^{\prime}$ add terms of higher order in $a$ so that the sum $F$ is exactly volume preserving. These higher order terms will not change our results, and are omitted in what follows.

\section{Normal hyperbolicity.}

As in [10], we invoque the theory of normal hyperbolicity of [6]. We start from the fact that $F_{0}$ is normally hyperbolic to the smooth fibration of $\mathbf{T}^{m+1}$ by circles $\{x\} \times \mathbf{T}$. 
Taking some $k \geq 2$ we apply [6] Theorems (7.1), (7.2). Thus we obtain a $\mathrm{C}^{1}$ neighborhood $U$ of $F_{0}$ in the $\mathrm{C}^{k}$ diffeomorphisms of $\mathbf{T}^{m+1}$ such that, for $F \in U$, there is an equivariant fibration $\pi: \mathbf{T}^{m+1} \rightarrow \mathbf{T}^{m}$ with

$$
\pi F=\Phi \pi
$$

The fibers $\pi^{-1}\{x\}$ are $\mathrm{C}^{k}$ circles forming a continuous fibration of $\mathbf{T}^{m+1}$ (this fibration is in general not smooth). Furthermore there is a $T F$-invariant continuous splitting of $T \mathbf{T}^{m+1}$ into three subbundles:

$$
T \mathbf{T}^{m+1}=E^{s}+E^{u}+E^{c}
$$

such that $E^{c}$ is 1-dimensional tangent to the circles $\pi^{-1}\{x\}, E^{s}$ is $m^{s}$-dimensional contracting and $E^{u}$ is $m^{u}$-dimensional expanding for $T F$.

If $\lambda_{r}<0$ (and $F$ is in a suitable $\mathrm{C}^{1}$-small neighborhood $U$ of $F_{0}$ ), we can introduce a continuous vector subbundle $E^{r}$ of $T \mathbf{T}^{m+1}$ which consists of vectors contracting under $T F^{n}$ faster than $\left(\lambda_{r}+\epsilon\right)^{n}$ where $\epsilon>0$ and $\lambda_{r}+\epsilon<\lambda_{r+1}$. In fact $E^{r}$ is a hyperbolic (attracting) fixed point for the action induced by $T F^{-1}$ on the bundle of $m_{1}+\ldots+m_{r}$ dimensional linear subspaces of $T \mathbf{T}^{m+1}$ (over $F^{-1}$ acting on $T^{m+1}$ ).

If $\lambda_{r}>0$, replacement of $F$ by $F^{-1}$ similarly yields a continuous subbundle $\bar{E}^{r}$ of $m_{r}+\ldots$ dimensional subspaces.

\section{Proposition.}

Assume that $F$ is of class $C^{k}, k \geq 2$, and that $F$ is $C^{k}$ close to $F_{0}$. The bundles $E^{r}$, $\bar{E}^{r}$, when restricted to a circle $\pi^{-1}\{x\}$ are of class $\mathrm{C}^{k-1}$, continuously in $x$.

If $\mathcal{G}$ denotes the (Grassmannian) manifold of $m_{1}+\ldots+m_{r}$ dimensional linear subspaces of $\mathbf{R}^{m+1}$, we may identify the bundle of $m_{1}+\ldots+m_{r}$ dinensional linear subspaces of $T \mathbf{T}^{m+1}$ with $\mathbf{T}^{m+1} \times \mathcal{G}$. We denote by $\mathcal{E} \in \mathcal{G}$ the spectral subspace of the matrix defining $\Phi$ corresponding to the smallest $m_{1}+\ldots+m_{r}$ eigenvalues (in absolute value, and repeated according to multiplicity).

If $\mathcal{F}_{0}$ is the action defined by $T F_{0}$ on $T \mathbf{T}^{m+1} \times \mathcal{G}$, the circles $\{x\} \times \mathbf{T} \times\{\mathcal{E}\}$ form an $\mathcal{F}_{0}$ invariant fibration of $\mathbf{T}^{m+1} \times\{\mathcal{E}\}$, to which $\mathcal{F}_{0}$ is normally hyperbolic. If $F$ is $\mathrm{C}^{k}$ close to $F_{0}$, the corresponding $\mathrm{C}^{k-1}$ action $\mathcal{F}$ is normally hyberbolic to a pertubed fibration where $\{x\} \times \mathbf{T} \times\{\mathcal{E}\}$ is replaced by $E^{r} \mid \pi^{-1}\{x\}$. According to [6] Theorem 7.4, Corollary (8.3) and the following Remark 2, we find that the $\mathrm{C}^{k-1}$ circle $E^{r} \mid \pi^{-1}\{x\} \subset \mathbf{T}^{m+1} \times \mathcal{G}$ depends continuously on $x \in \mathbf{T}^{m+1}$. Similarly for $\bar{E}$. [

Note that in [10], the $\mathrm{C}^{r}$ section theorem is used in a similar situation, giving estimates uniform in $x$. However, continuity in $x$ (not just uniformity) will be essential for us in what follows.

\section{Corollary.}

The splitting $T \mathbf{T}^{m+1}=E^{s}+E^{u}+E^{c}$ when restricted to a circle $\pi^{-1}\{x\}$ is of class $\mathrm{C}^{k-1}$, continuously in $x$. 
It is clear that $E^{c} \mid \pi^{-1}\{x\}$ is of class $\mathrm{C}^{k-1}$ because it is the tangent bundle to the $\mathrm{C}^{k}$ circle $\pi^{-1}\{x\}$. As to $E^{s}, E^{u}$, they are special cases of $E^{r}, \bar{E}^{r}$.

\section{Notation.}

Remember that $F=F_{0}+a F^{\prime}$, and fix $F^{\prime}$. We shall use the notation $\pi_{a}, E_{a}^{r}$, .. to indicate the $a$-dependence of $\pi, E^{r}, \ldots$

\section{Proposition.}

For small $\epsilon>0$ there is a continuous function $x \mapsto \gamma_{x}$ from $\mathbf{T}^{m}$ to $C^{k}(\mathbf{T} \times(-\epsilon, \epsilon) \rightarrow$ $\left.\mathbf{T}^{m}\right)$ such that $\gamma_{x}(y, 0)=0$ and $\pi_{a}^{-1}\{x\}=\left\{\left(x+\gamma_{x}(y, a), y\right): y \in \mathbf{T}\right\}$.

To see this define $\tilde{F}: \mathbf{T}^{m+1} \times(-\epsilon, \epsilon) \rightarrow \mathbf{T}^{m+1} \times(-\epsilon, \epsilon)$ by $\tilde{F}(\xi, a)=\left(\left(F_{0}+a F^{\prime}\right)(\xi), a\right)$ and observe that $\tilde{F}$ is normally hyperbolic to the 2 -dimensional manifolds

$$
\cup_{a \in(-\epsilon, \epsilon)}\left(\pi_{a}^{-1}\{x\}, a\right)
$$

and these are thus $\mathrm{C}^{k}$ 2-dimensional submanifolds of $\mathbf{T}^{m+1} \times(-\epsilon, \epsilon)$. $\square$

We may in the same manner replace $\pi_{a}^{-1}\{x\}$ by $\cup_{a \in(-\epsilon, \epsilon)}\left(\pi_{a}^{-1}\{x\}, a\right)$ in Proposition 3 and Corollary 4. Writing $E_{a}$ for $E_{a}^{r}, \bar{E}_{a}^{r}, E_{a}^{s}, E_{a}^{u}, E_{a}^{c}$, we obtain that $(\cdot, a) \mapsto E_{a}(\cdot)$, when restricted from $\mathbf{T}^{m+1} \times(-\epsilon, \epsilon)$ to $\cup_{a \in(-\epsilon, \epsilon)}\left(\pi_{a}^{-1}\{x\}, a\right)$ is of class $\mathrm{C}^{k-1}$. We rephrase this as follows:

\section{Proposition.}

The map

$$
x \mapsto\left\{(y, a) \mapsto E_{a}\left(x+\gamma_{x}(y, a), y\right)\right\}
$$

where $E_{a}$ stands for $E_{a}^{r}, \bar{E}_{a}^{r}, E_{a}^{s}, E_{a}^{u}, E_{a}^{c}$, is continuous $\mathbf{T}^{m} \rightarrow \mathrm{C}^{k-1}(\mathbf{T} \times(-\epsilon, \epsilon) \rightarrow$ Grassmannian of $\mathbf{R}^{m+1}$ ) where we have used the identification $T \mathbf{T}^{m+1}=\mathbf{T}^{m+1} \times \mathbf{R}^{m+1}$.

\section{Notation.}

From now on we write $E_{a}$ for $E_{a}^{r}, \bar{E}_{a}^{r}, E_{a}^{s}, E_{a}^{u}, E_{a}^{c}$. When $a=0, E_{0}$ is a trivial subbundle of $T \mathbf{T}^{m+1}=\mathbf{T}^{m+1} \times \mathbf{R}^{m+1}$, and we shall write $E_{0}=\mathbf{T}^{m+1} \times \mathcal{E}$, denoting thus by $\mathcal{E}$ a spectral subspace of the matrix on $\mathbf{R}^{m+1}$ defining $(\Phi, 1)$. We denote by $\mathcal{E}^{\perp}$ the complementary spectral subspace.

Taking $k=2$ we have then:

\section{Corollary.}

There are linear maps $G(x, y), R(x, y, a): \mathcal{E} \rightarrow \mathcal{E}^{\perp}$ such that $G(x, y)$ depends continuously on $(x, y) \in \mathbf{T}^{m} \times \mathbf{T}, R(x, y, a)$ on $(x, y, a) \in \mathbf{T}^{m} \times \mathbf{T} \times(-\epsilon, \epsilon)$,

$$
E_{a}\left(x+\gamma_{x}(y, a), y\right)=\{X+a G(x, y) X+R(x, y, a) X: X \in \mathcal{E}\}
$$

and $\|R(x, y, a)\|$ is o(a) uniformly in $x, y$. $\square$ 
Notice now that, if $\tilde{x}=\pi_{a}(x, y)$, then $x=\tilde{x}+\gamma_{\tilde{x}}(y, a)$, where $\gamma_{\tilde{x}}(y, a)=O(a)$. Now

$$
E_{a}(x, y)=E_{a}\left(\tilde{x}+\gamma_{\tilde{x}}(y, a), y\right)=\{X+a G(\tilde{x}, y) X+R(\tilde{x}, y, a) X: X \in \mathcal{E}\}
$$

differs from

$$
E_{a}\left(x+\gamma_{x}(y, a), y\right)=\{X+a G(x, y) X+R(x, y, a) X: X \in \mathcal{E}\}
$$

by the replacement $\tilde{x} \rightarrow x$ in the right-hand side, and $\operatorname{since} \operatorname{dist}(\tilde{x}, x)=O(a)$, we find that $\operatorname{dist}\left(E_{a}(x, y), E_{a}\left(x+\gamma_{x}(y, a), y\right)\right)=o(a)$. Therefore, changing the definition of $R$, we can again write:

\section{Corollary.}

There are linear maps $G(x, y), R(x, y, a): \mathcal{E} \rightarrow \mathcal{E}^{\perp}$, depending continuously on their arguments, such that

$$
E_{a}(x, y)=\{X+a G(x, y) X+R(x, y, a) X: X \in \mathcal{E}\}
$$

and $\|R(x, y, a)\|$ is $o(a)$ uniformly in $x, y$. $\square$

We may write $T_{\xi} F=T_{\xi}\left(F_{0}+a F^{\prime}\right)=D_{0}+a D^{\prime}(\xi)$ where $D_{0}$ does not depend on $\xi$ and preserves the decomposition $T_{\xi} M=\mathcal{E}+\mathcal{E}^{\perp}$. If we apply $T F$ to an element $X+a G X+R X$ of $E_{a}$ (as in Corollary 8) we obtain $X_{1}+$ element of $\mathcal{E}^{\perp} \in E_{a}$, with $X_{1} \in \mathcal{E}$ :

$$
X_{1}=D_{0} X+a D^{\prime} X+a^{2} D^{\prime} G X+a D^{\prime} R X \quad \text { projected on } \mathcal{E}
$$

Under $(T F)^{\wedge}$, the volume element $\theta$ in $E_{a}(\xi)$ is multiplied by a factor $M(\xi, a)$, and the projection in $\mathcal{E}$ of $(T F)^{\wedge} \theta$ is equal to the projection in $\mathcal{E}$ of $\theta$ multiplied by a factor $N(\xi, a)$ such that

$$
M(\xi, a)=N(\xi, a)+\ell_{a}(\xi)-\ell_{a}(F \xi)
$$

for suitable $\ell_{a}$. We may compute $N$ from (1):

$$
N(\xi, a)=N_{(0)}+a N_{(1)}(\xi)+a^{2} N_{(2)}(\xi)+o\left(a^{2}\right)
$$

To proceed we take now $E_{a}=E_{a}^{r}$, and assume $\lambda_{r}<0$. We have then, writing $d \xi$ for the volume element in $\mathrm{T}^{m+1}$,

$$
\begin{gathered}
L_{a}=\sum_{\ell=1}^{m_{1}+\ldots+m_{r}} \lambda_{a}^{(\ell)}=\int d \xi \log M(\xi, a)=\int d \xi \log N(\xi, a) \\
=L_{(0)}+a L_{(1)}(\xi)+a^{2} L_{(2)}(\xi)+o\left(a^{2}\right)
\end{gathered}
$$

More precisely, we shall prove 


\section{Proposition.}

If $\lambda_{r}<0$, we have

$$
\sum_{\ell=1}^{m_{1}+\ldots+m_{r}} \lambda_{a}^{(\ell)}=\sum_{k=1}^{r} m_{k} \lambda_{k}+a^{2} L+o\left(a^{2}\right)
$$

where

$$
L=\frac{1}{2} \sum_{n=-\infty}^{\infty} \int d \xi \operatorname{Tr}_{\mathcal{E}}\left(D_{0}^{-1} D^{\prime}(\xi)\right) \operatorname{Tr}_{\mathcal{E}}\left(D_{0}^{-1} D^{\prime}\left(F_{0}^{n} \xi\right)\right) \geq 0
$$

and $\mathcal{E}$ is the spectral subspace of the matrix defining $\Phi$ corresponding to the smallest $m_{1}+\ldots+m_{r}$ eigenvalues (in absolute value, and repeated according to multiplicity).

The proof that $L \geq 0$ is postponed to Remark $15(\mathrm{~b})$.

The proposition is obtained by comparing formula (2) with the formula (5) below, which we shall obtain by a second order perturbation calculation.

To first order in $a$ we have

$$
F^{n}=\left(F_{0}+a F^{\prime}\right)^{n}=F_{0}^{n}+a \sum_{j=1}^{n} F_{0}^{n-j} \circ F^{\prime} \circ F_{0}^{j-1}
$$

hence

$$
T_{\xi} F^{n}=D_{0}^{n}+a \sum_{j=1}^{n} D_{0}^{n-j} D^{\prime}\left(F^{j-1} \xi\right) D_{0}^{j-1}
$$

If we apply $T F^{n}$ to $X+a G X+R X \in E_{a}$ we obtain $X_{n}+$ element of $\mathcal{E}^{\perp} \in E_{a}$, with $X_{n} \in \mathcal{E}$. To zero-th order in $a, X_{n}=D_{0}^{n} X$, so we may write to first order $X_{n}=D_{0}^{n} X+a Y_{n}(\xi)$. Therefore, to first order in $a$,

$$
D_{0}^{n} X+a Y_{n}(\xi)+a G\left(F^{n} \xi\right) D_{0}^{n} X=D_{0}^{n} X+a \sum_{j=1}^{n} D_{0}^{n-j} D^{\prime}\left(F^{j-1} \xi\right) D_{0}^{j-1} X+a D_{0}^{n} G(\xi) X
$$

and, taking the components along $\mathcal{E}^{\perp}$

$$
G\left(F^{n} \xi\right) D_{0}^{n} X=\sum_{j=1}^{n} D_{0}^{n-j} D_{\perp}^{\prime}\left(F^{j-1} \xi\right) D_{0}^{j-1} X+D_{0}^{n} G(\xi) X
$$

where $D_{\perp}^{\prime}($.$) is D^{\prime}($.$) followed by taking the component along \mathcal{E}^{\perp}$, or

$$
\sum_{j=1}^{n} D_{0}^{-j} D_{\perp}^{\prime}\left(F^{j-1} \xi\right) D_{0}^{j-1} X+G(\xi) X=D_{0}^{-n} G\left(F^{n} \xi\right) D_{0}^{n} X
$$


When $n \rightarrow \infty$, the right-hand side tends to zero (exponentially fast, remember that $X \in \mathcal{E}$, $\left.G X \in \mathcal{E}^{\perp}\right)$. Therefore

$$
G(\xi) X=-\sum_{j=1}^{\infty} D_{0}^{-j} D_{\perp}^{\prime}\left(F^{j-1} \xi\right) D_{0}^{j-1} X
$$

which we shall use in the form

$$
G(\xi) X=-\sum_{n=0}^{\infty} D_{0}^{-n-1} D_{\perp}^{\prime}\left(F_{0}^{n} \xi\right) D_{0}^{n} X
$$

where we have written $F_{0}^{n}$ instead of $F^{n}$ since $G$ is evaluated to order 0 in $a$. (The right-hand side is an exponentially convergent series).

Returning to (1) we see that, to second order in $a$,

$$
\begin{aligned}
& X_{1}=D_{0} X+a D^{\prime}(\xi) X+a^{2} D^{\prime}(\xi) G(\xi) X \quad \text { projected on } \mathcal{E} \\
& =D_{0}\left(1+a D_{0}^{-1} D^{\prime}(\xi)+a^{2} D_{0}^{-1} D^{\prime}(\xi) G(\xi)\right) X \quad \text { projected on } \mathcal{E}
\end{aligned}
$$

Let now $\left(u^{(i)}\right)$ and $\left(u^{(i) \perp}\right)$ be conjugate bases of $\mathcal{E}$. Also let $\delta^{(i)}$ for $i=1, \ldots, m_{1}+\ldots+m_{r}$ be the eigenvalues of $D_{0}$ restricted to $\mathcal{E}$. Then, to second order in $a$,

$$
N(\xi, a) \wedge_{1}^{m_{1}+\ldots+m_{r}} u^{(\ell)}
$$

is, up to a factor of absolute value 1 ,

$$
\begin{gathered}
\left(\prod_{\ell=1}^{m_{1}+\ldots+m_{r}} \delta^{(\ell)}\right)\left[1+a \sum_{i=1}^{m_{1}+\ldots+m_{r}}\left(u^{(i) \perp}, D_{0}^{-1} D^{\prime}(\xi) u^{(i)}\right)\right. \\
+a^{2} \sum_{i<j}\left(\left(u^{(i) \perp}, D_{0}^{-1} D^{\prime}(\xi) u^{(i)}\right)\left(u^{(j) \perp}, D_{0}^{-1} D^{\prime}(\xi) u^{(j)}\right)\right. \\
\left.-\left(u^{(i) \perp}, D_{0}^{-1} D^{\prime}(\xi) u^{(j)}\right)\left(u^{(j) \perp}, D_{0}^{-1} D^{\prime}(\xi) u^{(i)}\right)+a^{2} \sum_{i}\left(u^{(i) \perp}, D_{0}^{-1} D^{\prime}(\xi) G(\xi) u^{(i)}\right)\right] \wedge_{\ell} u^{(\ell)}
\end{gathered}
$$

so that

$$
\begin{gathered}
N(\xi, a)=\left(\prod_{\ell=1}^{m_{1}+\ldots+m_{r}}\left|\delta^{(\ell)}\right|\right)\left[1+\left\{a \sum_{i}\left(u^{(i) \perp}, D_{0}^{-1} D^{\prime}(\xi) u^{(i)}\right)\right.\right. \\
+a^{2} \sum_{i<j}\left(\left(u^{(i) \perp}, D_{0}^{-1} D^{\prime}(\xi) u^{(i)}\right)\left(u^{(j) \perp}, D_{0}^{-1} D^{\prime}(\xi) u^{(j)}\right)\right. \\
\left.\left.\left.-\left(u^{(i) \perp}, D_{0}^{-1} D^{\prime}(\xi) u^{(j)}\right)\left(u^{(j) \perp}, D_{0}^{-1} D^{\prime}(\xi) u^{(i)}\right)\right)+a^{2} \sum_{i}\left(u^{(i) \perp}, D_{0}^{-1} D^{\prime}(\xi) G(\xi) u^{(i)}\right)\right\}\right]
\end{gathered}
$$


Since $\log \left|\delta^{(\ell)}\right|=\lambda_{0}^{(\ell)}$ we obtain, to second order in $a$,

$L_{a}=\int d \xi \log N(\xi, a)=m_{1} \lambda_{1}+\ldots+m_{r} \lambda_{r}+\int d \xi\left[\{\ldots\}-\frac{a^{2}}{2}\left(\sum_{i}\left(u^{(i) \perp}, D_{0}^{-1} D^{\prime}(\xi) u^{(i)}\right)\right)^{2}\right]$

where $\{\ldots\}$ has the same meaning as above. Write

$$
\Psi_{i}\left(\sum_{\ell} \xi_{\ell} u^{(\ell)}\right)=\left(u^{(i) \perp}, D_{0}^{-1} F^{\prime}\left(\sum_{\ell} \xi_{\ell} u^{(\ell)}\right)\right)
$$

The first term of $\int d \xi\{\ldots\}$ is

$$
a \sum_{i} \int d \xi\left(u^{(i) \perp}, D_{0}^{-1} T F^{\prime}(\xi) u^{(i)}\right)=a \sum_{i} \int d \xi \frac{\partial}{\partial \xi_{i}} \Psi_{i}
$$

which vanishes because $\int d \xi \frac{\partial}{\partial \xi_{i}} \ldots=0$. The next term in $\int d \xi\{\ldots\}$ is

$$
a^{2} \sum_{i<j} \int d \xi\left(\left(\frac{\partial \Psi_{i}}{\partial \xi_{i}}\right)\left(\frac{\partial \Psi_{j}}{\partial \xi_{j}}\right)-\left(\frac{\partial \Psi_{i}}{\partial \xi_{j}}\right)\left(\frac{\partial \Psi_{j}}{\partial \xi_{i}}\right)\right)=a^{2} \sum_{i<j} \int d \xi\left(\frac{\partial}{\partial \xi_{i}}\left(\Psi_{i} \frac{\partial \Psi_{j}}{\partial \xi_{j}}\right)-\frac{\partial}{\partial \xi_{j}}\left(\Psi_{i} \frac{\partial \Psi_{j}}{\partial \xi_{i}}\right)\right)
$$

which vanishes as above. Thus we are left with

$$
\begin{gathered}
L_{a}-\left(m_{1} \lambda_{1}+\ldots+m_{r} \lambda_{r}\right) \\
=a^{2} \int d \xi\left[\sum_{i}\left(u^{(i) \perp}, D_{0}^{-1} D^{\prime}(\xi) G(\xi) u^{(i)}\right)-\frac{1}{2}\left(\sum_{i}\left(u^{(i) \perp}, D_{0}^{-1} D^{\prime}(\xi) u^{(i)}\right)\right)^{2}\right]
\end{gathered}
$$

and we may write, using (3),

$$
\begin{gathered}
\sum_{i}\left(u^{(i) \perp}, D_{0}^{-1} D^{\prime}(\xi) G(\xi) u^{(i)}\right)=-\sum_{n=0}^{\infty} \sum_{i}\left(u^{(i) \perp}, D_{0}^{-1} D^{\prime}(\xi) D_{0}^{-n-1} D_{\perp}^{\prime}\left(F_{0}^{n}\right) D_{0}^{n} u^{(i)}\right) \\
=-\sum_{n=0}^{\infty} \sum_{i} \sum_{j}^{*}\left(u^{(i) \perp}, D_{0}^{-1} D^{\prime}(\xi) u^{(j)}\right)\left(u^{(j) \perp}, D_{0}^{-n-1} D^{\prime}\left(F_{0}^{n} \xi\right) D_{0}^{n} u^{(i)}\right)
\end{gathered}
$$

where we have introduces conjugate bases $\left(u^{(j)}\right),\left(u^{(j) \perp}\right)$ of $\mathcal{E}$, indexed by $j=m_{1}+\ldots+$ $m_{r}+1, \ldots, m+1$, and $\sum_{i}$ is over $i \leq m_{1}+\ldots+m_{r}+1, \sum_{j}^{*}$ is over $j>m_{1}+\ldots+m_{r}+1$. The above expression is also

$$
=-\sum_{n=0}^{\infty} \sum_{i} \sum_{j}^{*} \frac{\partial}{\partial \xi_{j}}\left(u^{(i) \perp}, D_{0}^{-1} F^{\prime}\left(\sum_{\ell} \xi_{\ell} u^{(\ell)}\right)\right) \frac{\partial}{\partial \xi_{i}}\left(u^{(j) \perp}, D_{0}^{-n-1} F^{\prime}\left(F_{0}^{n} \sum_{\ell} \xi_{\ell} u^{(\ell)}\right)\right)
$$

and integration by part gives thus

$$
\int d \xi \sum_{i}\left(u^{(i) \perp}, D_{0}^{-1} D^{\prime}(\xi) G(\xi) u^{(i)}\right)
$$




$$
\begin{gathered}
=-\sum_{n=0}^{\infty} \int d \xi \sum_{i} \frac{\partial}{\partial \xi_{i}}\left(u^{(i) \perp}, D_{0}^{-1} F^{\prime}\left(\sum_{\ell} \xi_{\ell} u^{(\ell)}\right)\right) \sum_{j}^{*} \frac{\partial}{\partial \xi_{j}}\left(u^{(j) \perp}, D_{0}^{-n-1} F^{\prime}\left(F_{0}^{n} \sum_{\ell} \xi_{\ell} u^{(\ell)}\right)\right) \\
=-\sum_{n=0}^{\infty} \int d \xi \operatorname{Tr}_{\mathcal{E}}\left(D_{0}^{-1} D^{\prime}(\xi)\right) \operatorname{Tr}_{\mathcal{E}^{\perp}}\left(D_{0}^{-n-1} D^{\prime}\left(F_{0}^{n} \xi\right) D_{0}^{n}\right) \\
=-\sum_{n=0}^{\infty} \int d \xi \operatorname{Tr}_{\mathcal{E}}\left(D_{0}^{-1} D^{\prime}(\xi)\right) \operatorname{Tr}_{\mathcal{E}}\left(D_{0}^{-1} D^{\prime}\left(F_{0}^{n} \xi\right)\right)
\end{gathered}
$$

The fact that $F=F_{0}+a F^{\prime}$ is volume preserving is expressed by $\operatorname{Tr}_{\mathbf{R}^{m+1}}\left(D_{0}^{-1} D^{\prime}(\xi)\right)=0$ hence

$$
\begin{gathered}
\int d \xi \sum_{i}\left(u^{(i) \perp}, D_{0}^{-1} D^{\prime}(\xi) G(\xi) u^{(i)}\right) \\
=\sum_{n=0}^{\infty} \int d \xi \operatorname{Tr}_{\mathcal{E}}\left(D_{0}^{-1} D^{\prime}(\xi)\right) \operatorname{Tr}_{\mathcal{E}}\left(D_{0}^{-1} D^{\prime}\left(F_{0}^{n} \xi\right)\right)
\end{gathered}
$$

and introducing this in (4) yields

$$
\begin{gathered}
L_{a}-\left(m_{1} \lambda_{1}+\ldots+m_{r} \lambda_{r}\right) \\
=a^{2}\left[\sum_{n=1}^{\infty} \int d \xi \operatorname{Tr}_{\mathcal{E}}\left(D_{0}^{-1} D^{\prime}(\xi)\right) \operatorname{Tr}_{\mathcal{E}}\left(D_{0}^{-1} D^{\prime}\left(F_{0}^{n} \xi\right)\right)+\frac{1}{2} \int d \xi\left(\operatorname{Tr}_{\mathcal{E}}\left(D_{0}^{-1} D^{\prime}(\xi)\right)\right)^{2}\right] \\
=\frac{a^{2}}{2} \sum_{n=-\infty}^{\infty} \int d \xi \operatorname{Tr}_{\mathcal{E}}\left(D_{0}^{-1} D^{\prime}(\xi)\right) \operatorname{Tr}_{\mathcal{E}}\left(D_{0}^{-1} D^{\prime}\left(F_{0}^{n} \xi\right)\right)
\end{gathered}
$$

where the last step used the invariance of $d \xi$ under $F_{0}^{n}$.

\section{Proof of Theorem 1.}

We use Proposition 9, the corresponding result with $F$ replaced by $F^{-1}$, and the fact that $\sum_{\ell=1}^{m} \lambda_{a}^{(\ell)}=0$ (because $F$ is volume preserving). This gives an estimate of all the sums of $\lambda_{a}^{(\ell)}$ that occur in Theorem 1 .

\section{Corollary.}

In the situation of Theorem 1, the central Lyapunov exponent is

$$
\begin{gathered}
\lambda^{c}=\frac{a^{2}}{2} \sum_{-\infty}^{\infty} \int d \xi\left[\operatorname{Tr}^{u}\left(D_{0}^{-1} D^{\prime}(\xi)\right) \operatorname{Tr}^{u}\left(D_{0}^{-1} D^{\prime}\left(F_{0}^{n} \xi\right)\right)-\operatorname{Tr}^{s}\left(D_{0}^{-1} D^{\prime}(\xi)\right) \operatorname{Tr}^{s}\left(D_{0}^{-1} D^{\prime}\left(F_{0}^{n} \xi\right)\right)\right] \\
=\frac{a^{2}}{2} \sum_{-\infty}^{\infty} \int d \xi\left[\operatorname{Tr}^{s}\left(D_{0}^{-1} D^{\prime}(\xi)\right)-\operatorname{Tr}^{u}\left(D_{0}^{-1} D^{\prime}(\xi)\right)\right] \operatorname{Tr}^{c}\left(D_{0}^{-1} D^{\prime}\left(F_{0}^{n} \xi\right)\right)
\end{gathered}
$$

where $\operatorname{Tr}^{s}, \operatorname{Tr}^{u}, \operatorname{Tr}^{c}$ denote the traces over the spectral subspaces $\mathcal{E}^{s}, \mathcal{E}^{u}, \mathcal{E}^{c}$ of $D_{0}$ corresponding to eigenvalues $<1,>1$, or $=1$ in absolute value ( $\mathcal{E}^{c}$ is one dimensional). 
Since $F$ preserves the volume, the sum of all Lyapunov exponents vanishes. Therefore $\lambda^{c}$ is minus the sum of the negative Lyapunov exponents, given by (5), minus the sum of the positive Lyapunov exponents. Note that replacing $F$ by $F^{-1}, \mathcal{E}^{s}$ by $\mathcal{E}^{u}$ (and, to the order considered, $D^{\prime}(\xi)$ by $\left.-D^{\prime}(\xi)\right)$ replaces the sum of the negative Lyapunov exponents by minus the sum of the positive exponents. This gives the first formula for $\lambda^{c}$.

To obtain the second formula, express $\operatorname{Tr}^{u} \operatorname{Tr}^{u}-\operatorname{Tr}^{s} \operatorname{Tr}^{s}$ in terms of $\operatorname{Tr}^{u} \pm \operatorname{Tr}^{s}$, and remember that (because $F$ preserves the volume) $\operatorname{Tr}^{s}+\operatorname{Tr}^{u}+\operatorname{Tr}^{c}=0$ when applied to $D_{0}^{-1} D^{\prime}(\xi)$.

The above formula (5) takes a particularly simple form in a special case described in the next theorem.

\section{Theorem.}

Let $\Phi$ be a hyperbolic automorphism of $\mathbf{T}^{m}$, with stable and unstable dimensions $m^{s}$ and $m^{u}=m-m^{s}$, and with entropy $\lambda_{0}^{u}$. Let $J: y \rightarrow y+\alpha(\bmod 1)$ be a translation of $\mathbf{T}$, and $\phi: \mathbf{T}^{m} \rightarrow \mathbf{T}$ a morphism $\neq 0$. Finally let $\psi: \mathbf{T} \rightarrow \mathbf{R}^{m}$ be a nullhomotopic $\mathrm{C}^{2}$ function.

Define $h, g_{a}: \mathbf{T}^{m} \times \mathbf{T} \rightarrow \mathbf{T}^{m} \times \mathbf{T}$ by

$$
h\left(\begin{array}{l}
x \\
y
\end{array}\right)=\left(\begin{array}{c}
\Phi x \\
J y+\phi \Phi x-\phi x
\end{array}\right) \quad, \quad g_{a}\left(\begin{array}{l}
x \\
y
\end{array}\right)=\left(\begin{array}{c}
x+a \psi(y)(\bmod 1) \\
y
\end{array}\right)
$$

and let $f_{a}=g_{a} \circ h$.

Denote by $\lambda_{a}^{s}$ (resp. $\lambda_{a}^{u}$ ) the sum of the smallest $m^{s}$ (resp. the largest $m^{u}$ ) Lyapunov exponents for $\left(f_{a}\right.$, volume). Also let $\lambda_{a}^{c}=-\lambda_{a}^{s}-\lambda_{a}^{u}$ be the "central exponent". Then $\lambda_{a}^{s}$, $\lambda_{a}^{u}, \lambda_{a}^{c}$ have expansions of order 2 in $a$ :

$$
\begin{gathered}
\lambda_{a}^{s}=-\lambda_{0}^{u}+\frac{a^{2}}{2} \int_{\mathbf{T}} d y\left((\nabla \phi) \psi^{\prime s}(y)\right)^{2}+o\left(a^{2}\right) \\
\lambda_{a}^{u}=\lambda_{0}^{u}-\frac{a^{2}}{2} \int_{\mathbf{T}} d y\left((\nabla \phi) \psi^{\prime u}(y)\right)^{2}+o\left(a^{2}\right) \\
\lambda_{a}^{c}=\frac{a^{2}}{2} \int_{\mathbf{T}} d y\left[\left((\nabla \phi) \psi^{\prime u}(y)\right)^{2}-\left((\nabla \phi) \psi^{\prime s}(y)\right)^{2}\right]+o\left(a^{2}\right)
\end{gathered}
$$

Here $\psi^{\prime s}(y)$ and $\psi^{\prime u}(y)$ are the components of the derivative $\psi^{\prime}(y) \in \mathbf{R}^{m}$ in the stable and unstable subspaces $\mathcal{E}^{s}$ and $\mathcal{E}^{u}$ for $\Phi$. Also, we have used $\nabla \phi: \mathbf{R}^{m} \rightarrow \mathbf{R}$ to denote the derivative of the map $\phi: \mathbf{T}^{m} \rightarrow \mathbf{T}$ with the obvious identifications.

This theorem is a simple (but nontrivial) extension of the result proved by Shub and Wilkinson [10]. In the situation that they consider $\Phi=\left(\begin{array}{ll}2 & 1 \\ 1 & 1\end{array}\right), J=$ identity, $\phi=(1,0)$, $\psi^{\prime}=\psi^{\prime u}$. [Remark that, in the notation of [10], $u_{0}=\left((1,1) \cdot v_{0}\right) /(m-1)=\left((1,0) \cdot v_{0}\right)$ so that the formula given in Proposition II of [10] agrees with our result above]. 


\section{Notation.}

We shall henceforth omit the $(\bmod 1)$. We shall keep $\nabla$ to denote the derivative in $\mathbf{T}^{m}$. With obvious abuses of notation, the reader may find it convenient to think of $\Phi$ or $\nabla \Phi$ as an $m \times m$ matrix (with integer entries and determinant \pm 1 ), and $\phi$ or $\nabla \phi$ as a row $m$-vector (with integer entries not all zero).

\section{Reformulation of the problem.}

Note that $f_{a}^{-1}=h^{-1} \circ g_{a}^{-1}$ where $h^{-1}, g_{a}^{-1}$ are obtained from $h, g_{a}$ by the replacements $\Phi, J, \phi, \psi \rightarrow \Phi^{-1}, J^{-1}, \phi,-\psi$. These replacements also interchange the stable and unstable subspaces for $\Phi$ and replace $\lambda^{s}, \lambda^{u}$ by $-\lambda^{u},-\lambda^{s}$. Therefore the formula for $\lambda^{u}$ in the theorem follows from the formula for $\lambda^{s}$. And the formula for $\lambda^{c}=-\lambda^{s}-\lambda^{u}$ also follows. To complete the proof of the theorem we turn now to the formula for $\lambda^{s}$.

Define

$$
\hat{\phi}\left(\begin{array}{l}
x \\
y
\end{array}\right)=\left(\begin{array}{c}
x \\
y+\phi x
\end{array}\right)
$$

then

$$
\begin{gathered}
F_{0}\left(\begin{array}{l}
x \\
y
\end{array}\right)=\hat{\phi}^{-1} h \hat{\phi}\left(\begin{array}{l}
x \\
y
\end{array}\right)=\left(\begin{array}{l}
\Phi x \\
J y
\end{array}\right) \\
\hat{g}_{a}\left(\begin{array}{l}
x \\
y
\end{array}\right)=\hat{\phi}^{-1} g_{a} \hat{\phi}\left(\begin{array}{l}
x \\
y
\end{array}\right)=\left(\begin{array}{c}
x+a \psi(y+\phi x) \\
y-a(\nabla \phi) \psi(y+\phi x)
\end{array}\right)
\end{gathered}
$$

so that

$$
F\left(\begin{array}{l}
x \\
y
\end{array}\right)=\hat{\phi}^{-1} f_{a} \hat{\phi}\left(\begin{array}{l}
x \\
y
\end{array}\right)=\hat{g}_{a} F_{0}\left(\begin{array}{l}
x \\
y
\end{array}\right)=\left(\begin{array}{c}
\Phi x+a \psi(J y+\phi \Phi x) \\
J y-a(\nabla \phi) \psi(J y+\phi \Phi x)
\end{array}\right)
$$

Finally, $F=F_{0}+a F^{\prime}$ with

$$
F_{0}\left(\begin{array}{l}
x \\
y
\end{array}\right)=\left(\begin{array}{l}
\Phi x \\
J y
\end{array}\right) \quad, \quad F^{\prime}\left(\begin{array}{l}
x \\
y
\end{array}\right)=\left(\begin{array}{c}
\psi(J y+\phi \Phi x) \\
-(\nabla \phi) \psi(J y+\phi \Phi x)
\end{array}\right)
$$

Since $F$ is conjugate (linearly) to $f_{a}$, we may compute $\lambda^{s}$ from $F$ instead of $f_{a}$.

\section{Proof of Theorem 12.}

Write $\mathbf{R}^{m+1}=\mathcal{E}^{s}+\mathcal{E}^{u}+\mathbf{R}$. We shall apply Proposition 9 with $\mathcal{E}=\mathcal{E}^{s}, \mathcal{E}^{\perp}=\mathcal{E}^{u}+\mathbf{R}$. Using $\xi=(x, y)$ and $X \in \mathcal{E}^{s}, Y \in \mathcal{E}^{u}, Z \in \mathbf{R}$ we may write

$$
\begin{gathered}
D_{0}\left(\begin{array}{c}
X+Y \\
Z
\end{array}\right)=\left(\begin{array}{c}
(\nabla \Phi)(X+Y) \\
Z
\end{array}\right) \\
D^{\prime}(\xi)\left(\begin{array}{c}
X+Y \\
Z
\end{array}\right)=\left(\begin{array}{c}
\psi^{\prime}(J y+\phi \Phi x)((\nabla \phi \Phi)(X+Y)+Z) \\
-(\nabla \phi) \psi^{\prime}(J y+\phi \Phi x)((\nabla \phi \Phi)(X+Y)+Z)
\end{array}\right)
\end{gathered}
$$

where $\psi^{\prime}$ denotes the derivative of $\psi$. Therefore

$$
\operatorname{Tr}_{\mathcal{E}}\left(D^{\prime}(\xi) D_{0}^{-1}\right)=(\nabla \phi) \psi^{\prime s}(J y+\phi \Phi x)
$$


and (5)contains the integrals

$$
\begin{gathered}
\int d \xi \operatorname{Tr}_{\mathcal{E}}\left(D_{0}^{-1} D^{\prime}(\xi)\right) \operatorname{Tr}_{\mathcal{E}}\left(D_{0}^{-1} D^{\prime}\left(F_{0}^{n} \xi\right)\right) \\
=\int d \xi\left[(\nabla \phi) \psi^{\prime s}(J y+\phi \Phi x)\right]\left[(\nabla \phi) \psi^{\prime s}\left(J^{n+1} y+\phi \Phi^{n+1} x\right)\right]
\end{gathered}
$$

Performing a change of variables $\bar{x}=\Phi x, \bar{y}=J y+\phi \Phi x$ we find that this is

$$
=\int d \bar{x} d \bar{y}\left[(\nabla \phi) \psi^{\prime s}(\bar{y})\right]\left[(\nabla \phi) \psi^{\prime s}\left(J^{n} \bar{y}+\phi \Phi^{n} \bar{x}-\phi \bar{x}\right)\right]
$$

We claim that this last integral vanishes unless $n=0$. This is because, if $n \neq 0$,

$$
\int d \bar{x} \psi^{\prime}\left(J^{n} \bar{y}+\phi \Phi^{n} \bar{x}-\phi \bar{x}\right)=0
$$

Indeed, $\phi \Phi^{n} \bar{x}-\phi \bar{x}$ is a linear combination with integer coefficients of the components $\bar{x}_{1}, \ldots, \bar{x}_{m}$ of $\bar{x}$, and the coefficients do not all vanish because $\phi \Phi^{n}=\phi$ is impossible ( $\Phi$ is hyperbolic and $\phi \neq 0$ ). Integrating the derivative $\psi^{\prime}$ with respect to a variable $\bar{x}_{j}$ really occuring in $\phi \Phi^{\ell} \bar{x}-\phi \bar{x}$ gives zero as announced.

Returning to (5) we have thus

$$
\begin{gathered}
\lambda_{a}^{s}+\lambda_{o}^{u}=\frac{a^{2}}{2} \int d \xi\left(\operatorname{Tr}_{\mathcal{E}}\left(D_{0}^{-1} D^{\prime}(\xi)\right)\right)^{2} \\
=\frac{a^{2}}{2} \int d \bar{y}\left((\nabla \phi) \psi^{\prime s}(\bar{y})\right)^{2}
\end{gathered}
$$

which is the formula given for $\lambda_{0}^{s}$ in Theorem 12. And according to Section 13 this completes our proof.

\section{Final remarks.}

(a) Shub and Wilkinson [10] showed that close to a diffeomorphism (hyperbolic automorphism $\Phi$ of $\left.\mathbf{T}^{2}\right) \times\left(\right.$ identity on $\mathbf{T}$ ) there is a $\mathrm{C}^{1}$ open set of ergodic volume preserving $\mathrm{C}^{2}$ diffeomorphisms of $\mathbf{T}^{3}$ with central Lyapunov exponent $\lambda^{c}>0$. They remark that their result extends to the situation where $\Phi$ is a hyperbolic automorphism of $\mathbf{T}^{m}$ with onedimensional expanding eigenspace. More generally, if $\Phi$ is any hyperbolic automorphism of $\mathbf{T}^{m}$, Theorem 12 gives close to $(\Phi$, rotation of $\mathbf{T})$ in $\mathrm{C}^{2}\left(\mathbf{T}^{m+1}\right)$ a diffeomorphism $F$ with $\lambda^{c}>0$. Since $\lambda^{c}$ is given by an integral over the volume of a local "central" stretching exponent, we have $\lambda^{c}>0$ in a $\mathrm{C}^{1}$ neighborhood of $F$. But by a result of Dolgopyat and Wilkinson [5] (Corollary 0.5), stable ergodicity is here $\mathrm{C}^{1}$ open and dense in the $\mathrm{C}^{2}$ volume preserving diffeomorphisms: we have center bunching and stable dynamical coherence because we consider perturbations of $(\Phi$, rotation of $\mathbf{T})$ for which the center foliation is $\mathrm{C}^{1}$, see [6], [7]. In conclusion, close to (hyperbolic automorphism $\Phi$ of $\left.\mathbf{T}^{m}\right) \times($ rotation on $\mathbf{T})$ there is a $\mathrm{C}^{1}$ open set $V$ of ergodic volume preserving $\mathrm{C}^{2}$ diffeomorphisms of $\mathbf{T}^{m+1}$ with 
central Lyapunov exponent $\lambda^{c}>0$ (or also with $\lambda^{c}<0$ ). In particular, if $F \in V$, the conditional measures of the volume on the circles $\pi^{-1}\{x\}$ are atomic, as discussed in [9].

(b) The coefficient $L$ in Proposition 9 is $\geq 0$. Consider indeed the unitary operator $U$ defined by $U \psi=\psi \circ F$ on $L^{2}\left(\mathbf{T}^{m+1}\right.$, volume), and let $E($.$) be the corresponding spectral$ measure, so that

$$
U=\int_{\mathbf{T}} e^{2 \pi i \theta} E(d \theta)
$$

If $\psi(\xi)=\operatorname{Tr}_{\mathcal{E}}\left(D_{0}^{-1} D^{\prime}(\xi)\right)$ we have a measure $\nu \geq 0$ on $\mathbf{T}$ defined by $\nu(d \theta)=(\psi, E(d \theta) \psi)$ and the Fourier coefficients

$$
c_{n}=\int e^{2 \pi n i \theta} \nu(d \theta)=\int d \xi \operatorname{Tr}_{\mathcal{E}}\left(D_{0}^{-1} D^{\prime}(\xi)\right)\left(D_{0}^{-1} D^{\prime}\left(F_{0}^{n} \xi\right)\right)
$$

of this measure tend to zero exponentially. Therefore $\nu(d \theta)=\rho(\theta) d \theta$ has a smooth density $\rho$ and

$$
L=\frac{1}{2} \sum_{n=-\infty}^{\infty} c_{n}=\frac{1}{2} \rho(0) \geq 0
$$

(c) Suppose now that $F$ is not necessarily a volume preserving perturbation of $F_{0}$. We may still hope that $F$ has an SRB measure $\rho_{a}$. If $F_{0}$ were hyperbolic, we would have an expansion

$$
\rho_{a}=\rho_{0}+a \delta+o(a)
$$

(see [8]) with $\rho_{0}=$ Lebesgue measure and $\delta$ a distribution. For smooth $\Psi, \delta(\Psi)$ is given (because $\rho_{0}$ is Lebesgue measure) by the simple formula (see [8])

$$
\delta(\Psi)=-\sum_{0}^{\infty} \rho_{0}\left(\left(\Psi \circ F_{0}^{n}\right) \cdot \operatorname{div}\left(F^{\prime} \circ F_{0}^{-1}\right)\right.
$$

Similarly (replacing $F$ by $F^{-1}$, hence $F_{0}, D_{0}^{-1} D^{\prime}(\xi)$ by $F_{0}^{-1},-D^{\prime}\left(F_{0}^{-1} \xi\right) D_{0}^{-1}$ we see that the anti-SRB state has an expansion

$$
\bar{\rho}_{a}=\rho_{0}+a \bar{\delta}+o(a)
$$

with

$$
\begin{aligned}
\bar{\delta}(\Psi) & =\sum_{n=1}^{\infty} \int d \xi \Psi\left(F_{0}^{-n} \xi\right) \operatorname{Tr}_{\mathbf{R}^{m+1}}\left(D^{\prime}\left(F_{0}^{-1} \xi\right) D_{0}^{-1}\right) \\
& =\sum_{n=0}^{\infty} \int d \xi \Psi\left(F_{0}^{-n} \xi\right) \operatorname{Tr}_{\mathbf{R}^{m+1}}\left(D_{0}^{-1} D^{\prime}(\xi)\right)
\end{aligned}
$$

We can now estimate the Lyapunov exponents for $\left(F, \rho_{a}\right)$ to second order in $a$ even though we are not sure of the existence of the SRB measure $\rho_{a}$. We simply assume that we can use the formula for $\delta(\Psi)$. Going through the proof of Proposition 9 we have to replace 
$\int d \xi \log N(\xi, a)$ by $\rho_{a}(\log N(., a))$ and (to second order in $a$ ) this adds to the right-hand side of (4) a term

$$
-a^{2} \sum_{n=1}^{\infty} \int d \xi \operatorname{Tr}_{\mathcal{E}}\left(D_{0}^{-1} D^{\prime}(\xi)\right) \operatorname{Tr}_{\mathbf{R}^{m+1}}\left(D_{0}^{-1} D^{\prime}(\xi)\right)
$$

Taking into account the integrations by part we obtain now instead of (5) the formula

$$
\begin{gathered}
L_{a}-\left(m_{1} \lambda_{1}+\ldots+m_{r} \lambda_{r}\right)=\frac{a^{2}}{2} \sum_{n=-\infty}^{\infty} \int d \xi \operatorname{Tr}_{\mathcal{E}}\left(D_{0}^{-1} D^{\prime}(\xi)\right) \operatorname{Tr}_{\mathcal{E}}\left(D_{0}^{-1} D^{\prime}\left(F_{0}^{n} \xi\right)\right) \\
-a^{2} \sum_{n=-\infty}^{\infty} \int d \xi \operatorname{Tr}_{\mathcal{E}}\left(D_{0}^{-1} D^{\prime}(\xi)\right) \operatorname{Tr}_{\mathbf{R}^{m+1}}\left(D_{0}^{-1} D^{\prime}\left(F_{0}^{n} \xi\right)\right)
\end{gathered}
$$

Let $a^{2} L^{s}, a^{2} L^{u}, a^{2} L^{c}$ be the $a^{2}$ contributions to the sum of the noncentral negative, noncentral positive, and the central Lyapunov exponents for the SRB measure. We obtain $a^{2} L^{s}$ from (6) when $n_{r}=n^{s}$. A similar calculation gives $a^{2} L^{u}$ (it is convenient here to work via the anti-SRB measure, then replace $F$ by $F^{-1}$ ). Estimating the average expansion coefficient gives $a^{2}\left(L^{s}+L^{u}+L^{c}\right)=\rho_{a}\left(\log \operatorname{det}\left(D_{0}+a D^{\prime}().\right)\right.$, hence $L^{s}+L^{u}+L^{c}$, hence $L^{c}$. The results are

$$
\begin{aligned}
L^{s} & =\frac{1}{2} \sum_{n=-\infty}^{\infty} \int d \xi \operatorname{Tr}^{s}\left(D_{0}^{-1} D^{\prime}(\xi)\right) \operatorname{Tr}^{s}\left(D_{0}^{-1} D^{\prime}\left(F_{0}^{n} \xi\right)\right) \\
- & \sum_{n=-\infty}^{\infty} \int d \xi \operatorname{Tr}^{s}\left(D_{0}^{-1} D^{\prime}(\xi)\right) \operatorname{Tr}_{\mathbf{R}^{m+1}}\left(D_{0}^{-1} D^{\prime}\left(F_{0}^{n} \xi\right)\right) \\
L^{u}= & -\frac{1}{2} \sum_{n=-\infty}^{\infty} \int d \xi \operatorname{Tr}^{u}\left(D_{0}^{-1} D^{\prime}(\xi)\right) \operatorname{Tr}^{u}\left(D_{0}^{-1} D^{\prime}\left(F_{0}^{n} \xi\right)\right) \\
L^{c}= & -\frac{1}{2} \sum_{n=-\infty}^{\infty} \int d \xi \operatorname{Tr}^{c}\left(D_{0}^{-1} D^{\prime}(\xi)\right) \operatorname{Tr}^{c}\left(D_{0}^{-1} D^{\prime}\left(F_{0}^{n} \xi\right)\right) \\
& -\sum_{n=-\infty}^{\infty} \int d \xi \operatorname{Tr}^{c}\left(D_{0}^{-1} D^{\prime}(\xi)\right) \operatorname{Tr}^{u}\left(D_{0}^{-1} D^{\prime}\left(F_{0}^{n} \xi\right)\right) \\
L^{s}+L^{u}+L^{c} & =-\frac{1}{2} \sum_{n=-\infty}^{\infty} \int d \xi \operatorname{Tr}_{\mathbf{R}^{m+1}}\left(D_{0}^{-1} D^{\prime}(\xi)\right) \operatorname{Tr}_{\mathbf{R}^{m+1}}\left(D_{0}^{-1} D^{\prime}\left(F_{0}^{n} \xi\right)\right)
\end{aligned}
$$

which can be rewritten variously.

The existence of second order expansions for the Lyapunov exponents gives added interest to the question whether the SRB measure $\rho_{a}$ really exists for (small) finite $a$.

(d) The author has not looked seriously into possible extensions of the results presented here. Generalizations are thus left for the reader to formulate, and to prove. 


\section{References.}

[1] A. Avila and J. Bochi. "A formula with some applications to the theory of Lyapunov exponents." Preprint

[2] C. Bonatti, X. Gómez-Mont and M. Viana. "Généricité d'expoants de Lyapunov non-nuls des produits déterministes de matrices." Preprint

[3] K. Burns and A. Wilkinson. "Stable ergodicity of skew products." Ann. Sci. Ecole Norm. Sup. 32,859-889(1999).

[4] D. Dolgopyat. "On differentiability of SRB states." Preprint.

[5] D. Dolgopyat and A. Wilkinson. "Stable accessibility is $\mathrm{C}^{1}$ dense." Preprint

[6] M. Hirsch, C.C. Pugh, and M. Shub. Invariant manifolds. Lect. Notes in Math. 583 Springer, Berlin, 1977.

[7] C. Pugh, and M. Shub. "Stable ergodicity and julienne quasi-conformality." J. Eur. Math. Soc. 2,1-52(2000).

[8] D. Ruelle. "Differentiation of SRB states." Commun. Math. Phys. 187,227241(1997).

[9] D. Ruelle and A. Wilkinson. "Absolutely singular dynamical foliations." Commun. Math. Phys., to appear.

[10] M. Shub and A. Wilkinson. "Pathological foliations and removable exponents." Inventiones Math. 139,495-508(2000). 